\title{
Posterior scleritis mimicking orbital cellulitis
}

\author{
Michael Rossiter-Thornton, Lia Rossiter-Thornton, Raf Ghabrial and Domit A Azar
}

\section{Clinical record}

In 2008, an 88-year-old woman with a background of well controlled systemic hypertension and high myopia presented to an emergency department with a 5-day history of left periorbital swelling, erythema and mild discomfort, without visual loss. She was seen in the ophthalmology clinic on the same day. Her visual acuity was $6 / 12$ in each eye. She was afebrile, with left upper and lower lid erythema, oedema closing the palpebral fissure, and conjunctival and scleral injection. Slit-lamp biomicroscopy revealed normal anterior chambers; clear, well centred intraocular lens implants from previous uncomplicated cataract surgery; and posterior staphylomata (an incidental finding of bulging of the globe seen in some highly myopic patients). A computed tomography (CT) scan revealed left periorbital soft tissue thickening, with stranding confined to the preseptal area (Figure A). Radiologically, the patient appeared to have periorbital cellulitis, although clinical examination, being more sensitive to ocular signs, revealed postorbital septal spread of infection as evidenced by scleral injection (not expected to be seen on a CT scan), leading to the diagnosis of orbital cellulitis. Intravenous antibiotics were administered for 3 days, with moderate resolution of the periorbital swelling, although the scleral injection remained.

Three days after discharge on oral antibiotics, the patient re-presented to the emergency department with recurrence of left periorbital swelling, erythema and discomfort. After administration of intravenous antibiotics for five days, her lid oedema and erythema resolved, but conjunctival and scleral injection persisted. Investigations during this second admission revealed raised erythrocyte sedimentation rate (ESR) and $C$ reactive protein (CRP) level, with a normal blood leucocyte count.

Soon after her second discharge, the patient presented again with persisting and worsening erythema of the left eye, with no associated lid swelling or erythema. A clinical diagnosis of non-necrotising anterior scleritis was made (Figure B), and it was thought that conjunctival lymphoma should be excluded. B-scan ultrasonography revealed fluid in Tenon's capsule and scleral thickening, consistent with anterior and posterior scleritis (Figure B), and investigations again revealed a raised ESR and CRP level. Screening for tuberculosis (TB) (Mantoux test and chest x-ray), antinuclear antibodies, extractable nuclear antigen antibodies, rheumatoid factor, syphilis, and angiotensin-converting enzyme (an indicator of sarcoidosis) were negative. Screening for Wegener's granulomatosis with perinuclear antineutrophil cytoplasmic antibodies was positive, but attempted confirmation by screening for antiproteinase 3 antibodies and antimyeloperoxidase antibodies was negative. Magnetic resonance imaging revealed marked high $\mathrm{T} 2$ signalling and enhancement of the left sclera, extraocular muscle insertions and distal optic nerve (Figure C). A scleroconjunctival biopsy was performed to exclude conjunctival lymphoma or other uncommon diagnoses, such as tuberculosis and fungal infection. The biopsy revealed a dense inflammatory infiltrate consisting of B and T lymphocytes, plasma cells and occasional neutrophils, with no granulomatous inflammation. Flow cytometry; immunohistochemistry; bacterial, fungal and mycobacterial staining and culture; and TB polymerase chain reaction tests were all negative. Haematological consultation excluded systemic or central nervous system lymphoma.

A short course of oral prednisolone ( $1 \mathrm{mg} / \mathrm{kg} /$ day for 14 days) resulted in complete resolution of the patient's signs and symptoms, and she remained well at 6-week follow-up.

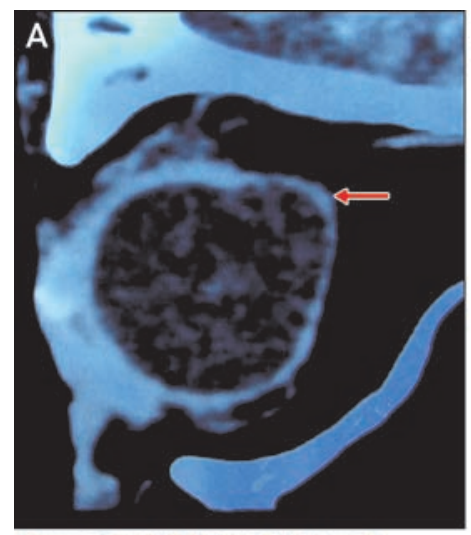

A: Sagittal computed tomography scan showing left periorbital soft tissue thickening and posterior staphyloma.

B: Superior scleral injection and B-scan ultrasound showing fluid in Tenon's capsule and scleral thickening, consistent with anterior and posterior scleritis.

C: Magnetic resonance images showing high $\mathrm{T} 2$ signalling and enhancement of the left sclera, extraocular muscle insertions, and distal optic nerve.
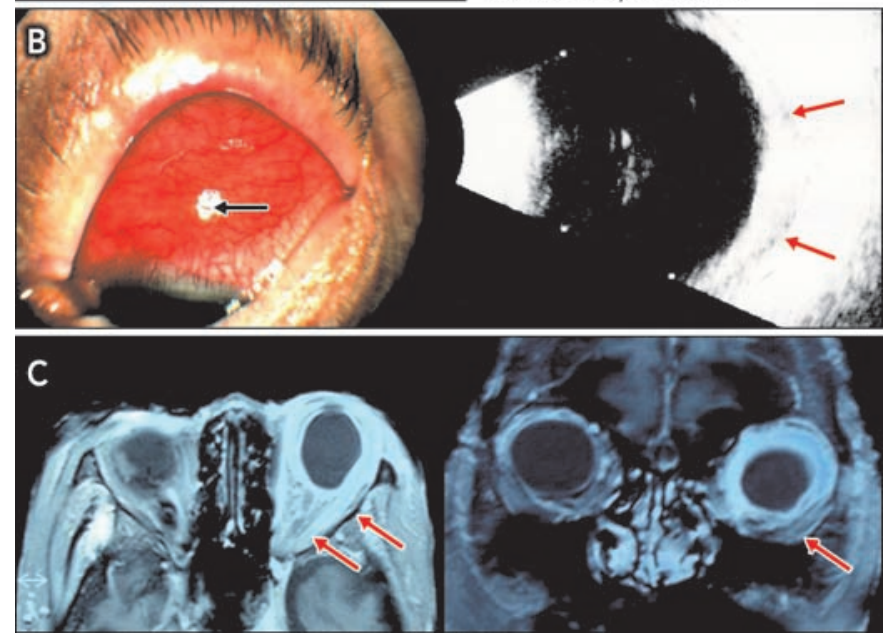

$\mathrm{T}$ o our knowledge, this is only the second reported case of posterior scleritis mimicking orbital cellulitis. We believe that a diagnosis of posterior scleritis should always be considered when patients with presumed periorbital or orbital cellulitis do not respond appropriately to intravenous antibiotic treatment.

In the previous report of posterior scleritis mimicking orbital cellulitis, the authors hypothesised that inflammation had spread anteriorly, involving the upper lid structures, causing lid swelling and simulating cellulitis. ${ }^{1}$

Orbital cellulitis has an incidence of 5.8 per 100000 population in people aged 65 years or older. ${ }^{2}$ Posterior scleritis is much less common. McCluskey and colleagues ${ }^{3}$ published a singlecentre case series of patients with posterior scleritis seen at Moorfields Eye Hospital in London that shed much light on the nature of the disease. Between 1974 and 1996, only 137 patients were diagnosed with posterior scleritis. Interestingly, in this series, only $31 \%$ of patients had visual loss, little more than half (56\%) had pain, and $17 \%$ had no physical signs of posterior scleritis on examination. Anterior scleritis at or after presentation occurred in $60 \%$ of patients, with $36 \%$ having combined anterior and posterior scleritis on presentation. Only $28 \%$ had an associated systemic disease, and no patient had necrotising posterior scleritis. 
Our patient had no pain, no visual loss, and no physical sign of posterior scleritis on clinical examination. Her initial examination appeared to be consistent with the diagnosis of orbital cellulitis. With respect to diagnostic criteria, there were many signs in her clinical presentation that are shared by orbital cellulitis and posterior scleritis. Features that favoured the eventual diagnosis of posterior scleritis were the presence of physical and radiological signs in the absence of an appropriate response to intravenous antibiotic treatment, and a positive response to oral prednisolone treatment.

Patients presenting with signs of periorbital or orbital cellulitis should always be treated with intravenous antibiotics immediately, pending the results of any radiological investigation. However, when a systemically well patient with no radiological or clinical evidence of an orbital abscess requiring surgical drainage does not respond positively to intravenous antibiotic treatment, ophthalmologists should suspect non-infective aetiology.

\section{Competing interests}

None identified.

\section{Author details}

Michael Rossiter-Thornton, BMedSc(Hons), MB BS(Hons), Resident Medical Officer ${ }^{1}$

Lia Rossiter-Thornton, BMedSc, MB BS(Hons), Resident Medical Officer $^{1}$

Raf Ghabrial, MB BS, FRANZCO, Ophthalmic Surgeon ${ }^{1}$ and Clinical Senior Lecturer ${ }^{2}$

Domit A Azar, MB BS(Hons), MPH(Hons), FRANZCO, Ophthalmic Surgeon ${ }^{1}$ and Clinical Senior Lecturer ${ }^{2}$

\section{Lessons from practice}

- Periorbital cellulitis can spread posteriorly into the orbit, cavernous sinus and brain, and is therefore potentially lethal.

- Intravenous antibiotic treatment for probable cellulitis should be started immediately, without waiting for the results of diagnostic radiological investigations.

- When a systemically well patient with no radiological or clinical evidence of an orbital abscess requiring surgical drainage does not respond positively to intravenous antibiotic treatment, ophthalmologists should suspect non-infective aetiology.

- Any patient with periorbital or orbital cellulitis should be assessed by an ophthalmologist.

1 Department of Ophthalmology, Concord Repatriation General Hospital, Sydney, NSW.

2 Sydney Medical School, University of Sydney, Sydney, NSW.

Correspondence: domit.azar@sydney.edu.au

\section{References}

1 Rubinstein A, Riddell C. Posterior scleritis mimicking orbital cellulitis [letter]. Eye 2005; 19: 1232-1233.

2 Soroudi A, Casey R, Pan D, Baker R. An epidemiologic survey of orbital cellulitis. Invest Ophthalmol Vis Sci 2003; 44: e-abstract 786. http:// abstracts.iovs.org/cgi/content/abstract/44/5/786 (accessed Jul 2010).

3 McCluskey P, Watson P, Lightman S, et al. Posterior scleritis. Clinical features, systemic associations, and outcomes in a large series of patients. Ophthalmology 1999; 106: 2380-2386.

(Received 21 Oct 2009, accepted 4 Jun 2010) 